\title{
An Investigation of Factors Associated With Paranoid Ideation among a Sample of Regular Ecstasy Consumers
}

\author{
A.J. Matthews* and R. Bruno \\ School of Psychology, University of Tasmania, Australia
}

Keywords: Ecstasy, MDMA, paranoia, paranoid ideation, risk factors.

\section{INTRODUCTION}

Paranoid ideation (thought) can be characterised by exaggerated self-referential biases and a pervasive and exaggerated view of the social world as threatening and untrustworthy [1]. Previous research suggests the use of drugs such as methamphetamine, cannabis, and ecstasy may be associated with the experience of clinical levels of paranoid ideation $[2,3]$.

Regular ecstasy users show significantly higher levels of self-rated paranoid ideation (SCL-90-R) relative to matched controls [2]. However, regular ecstasy consumers are typically polydrug users and there is evidence that psychopathological symptoms experienced by this group are more strongly associated with the use of other substances, such as cannabis, rather than being solely reflective of ecstasy use [2]. The aim of the present research was to investigate correlates of sub-clinical paranoid ideation among a sample of regular ecstasy consumers.

\section{METHODS}

200 regular (at least monthly) ecstasy users were interviewed for the 2007 and 2008 Ecstasy and Related Drug Reporting System (EDRS) in Tasmania [4, 5]. The project examines trends in ecstasy and related drugs (cannabis, methamphetamine, cocaine, LSD, ketamine, MDA, GHB) and associated risk behaviours and health-related harms among regular ecstasy consumers in Australian capital cities. Paranoid ideation was assessed using a Paranoia Scale (PS) developed for use in sub-clinical populations [1]. Subgroups with high $(\geq 30)$ and low $(<30)$ scores on the PS were identified and step-wise forwards logistic regression was used to examine the significant correlates of high scores.

\section{RESULTS}

Based on theory and preliminary analyses, the following factors were included in the regression analysis: sex, age of onset of ecstasy use, frequency of ecstasy use, recent use of cannabis, recent use of LSD, frequency of cannabis use, and frequency of methamphetamine use. The resulting, significant regression equation accounted for $29 \%$ of variance in the prediction of high PS scores and correctly

*Address correspondence to this author at the School of Psychology, University of Tasmania, Private Bag 30, Hobart, 7001, Australia; Tel: +61 (3) 6226 2945; Fax: +61 (3) 622622883 ;

E-mail: Allison.Matthews@utas.edu.au predicted $68 \%$ of total cases and $77 \%$ of risk cases $\chi^{2}=24.70$, $p>.001$. Recent use of cannabis was the most substantial predictor of high PS scores accounting for $12.5 \%$ of unique variance $(\mathrm{OR}=4.04,95 \% \mathrm{CI}=1.39-11.75)$. High frequency ecstasy use and early age of onset for ecstasy also made significant independent contributions accounting for $10 \%$ $(\mathrm{OR}=1.08,95 \% \mathrm{CI}=1.01-1.14)$ and $7 \%(\mathrm{OR}=1.31,95 \%$ $\mathrm{CI}=1.03-1.67)$ of variance respectively.

\section{DISCUSSION}

Consistent with previous findings [2] recent use of cannabis was the most substantial predictor of high PS scores. However, early onset of ecstasy use and high frequency ecstasy use were also independently associated with high levels of paranoid ideation in this population. High PS scores were also associated with methamphetamine use, though this did not contribute unique variance to the model.

There was an association between high PS scores and self-reported mental health problems (in particular anxiety and depression), and associated help-seeking, as well as high levels of psychological distress. Paranoid ideation is associated with various other constructs including anxiety, depression, anger, self esteem and interpersonal trust $[1,6]$, all of which may have contributed to the present results. It is not possible to determine the causative nature of the relationships reported in the present study, as drug use may contribute to the development of paranoid ideation and vice versa. While previous research suggests that cannabis may play a causative role in the development of psychotic symptoms, further longitudinal research is required to investigate this association for ecstasy.

\section{CONCLUSIONS}

The present findings suggest an association between paranoid ideation and use of ecstasy, cannabis, and methamphetamine among regular ecstasy users. High levels of paranoid ideation were also associated with elevated selfreported mental health problems and psychological distress. While further longitudinal research is required, paranoid ideation may be a risk factor for, or a consequence of drug use in this population.

\section{REFERENCES}

[1] Fenigstein A, Vanable PA. Paranoia and self-consciousness. J Pers Soc Psychol 1992; 62: 129-38.

[2] Daumann J, Hensen G, Thimm B, Rezk M, Till B, GouzoulisMayfrank E. Self-reported psychopathological symptoms in 
recreational ecstasy (MDMA) users are mainly associated with regular cannabis use: further evidence from a combined crosssectional/longitudinal investigation. Psychopharmacology 2004; 173: $398-404$.

[3] Johns LC, Cannon M, Singleton N, et al. Prevalence and correlates of self-reported psychotic symptoms in the British population. Br J Psychiatry 2004; 185: 298-305.

[4] Matthews A, Bruno R. Tasmanian Trends in Ecstasy and Related Drug Markets 2007: findings from the Ecstasy and Related Drugs Reporting System (EDRS). Australian Drug Trends Series No. 14.
Sydney: National Drug and Alcohol Research Centre, University of New South Wales 2008 .

[5] Matthews A, Bruno R. Tasmanian Trends in Ecstasy and Related Drug Markets 2008: findings from the Ecstasy and Related Drugs Reporting System (EDRS). Australian Drug Trends Series No. 32. Sydney: National Drug and Alcohol Research Centre, University of New South Wales 2009.

[6] Combs DR, Penn DL, Fenigstein A. Ethnic differences in subclinical paranoia. Cultur Divers Ethnic Minor Psychol 2002; 8 : 248-56.

(C) Matthews and Bruno; Licensee Bentham Open.

This is an open access article licensed under the terms of the Creative Commons Attribution Non-Commercial License (http://creativecommons.org/licenses/ by-nc/3.0/) which permits unrestricted, non-commercial use, distribution and reproduction in any medium, provided the work is properly cited. 